\title{
LDPC-Coded Optical Communication over the Atmospheric Turbulence Channel
}

\author{
Ivan B. Djordjevic \\ Department of Electrical and Computer Engineering, University of Arizona \\ Tucson, AZ 85721, USA \\ ivan@ece.arizona.edu
}

\begin{abstract}
The free-space optical (FSO) systems that are robust in the presence of atmospheric turbulence are discussed in this invited paper: (i) coded orthogonal frequency division multiplexing (OFDM), and (ii) coded multiple-input multipleoutput (i.e., multi-laser multi-detector or MIMO) concept; both employing low-density parity-check (LDPC) codes. Two MIMO concepts are discussed: (i) repetition-MIMO, and (ii) space-time coded-MIMO. The second goal of this paper is to discuss the incompatibility that arises from the bandwidth mismatch between RF/microwave and optical channels. We describe two coded modulation schemes suitable for hybrid microwave-optical communications: (i) coded-OFDM as multiplexing technique, and (ii) Q-ary bit-interleaved coded pulse-amplitude modulation. The third goal of this paper is to describe the ultimate channel capacity limits, and to see how close we can approach those limits with proposed coded-MIMO concepts.
\end{abstract}

Keywords- Free-space optical (FSO) communications; atmospheric turbulence; orhtogonal frequency division multiplexing (OFDM); multiple-input multiple output (MIMO), space-time coding; low-density parity-check (LDPC) codes; direct detection

\section{INTRODUCTION}

Free-space optical (FSO) communications have received significant attention recently, as a possible alternative to solve the bottleneck connectivity problem, and as a supplement to conventional RF/microwave links [1]. However, an optical wave propagating trough the air experiences fluctuations in amplitude and phase due to atmospheric turbulence, also known as scintillation.

The incompatibility of $\mathrm{RF} / \mathrm{microwave}$ and optical communication technologies arises from the large bandwidth mismatch between these two channel types and is now widely believed to be the limiting factor in efforts to further increase future transport capabilities.

The purpose of this invited paper, based on our several recent publications [2]-[5], is twofold: (i) to study different scenarios capable of operating under the strong atmospheric turbulence, and (ii) to study different solutions to bandwidth mismatch problem or alternatively to study different scenarios suitable for hybrid microwave-optical communications.

This work was supported in part by the National Science Foundation (NSF) under Grant IHCS 0725405.
To deal with atmospheric turbulence we propose to use either: (i) coded-orthogonal frequency division multiplexing (OFDM) scenario, or (ii) coded-multiple-input multiple output (MIMO) scenario. The coding in both scenarios is based on the best known codes-low-density parity-check (LDPC) codes.

To solve the $\mathrm{RF} /$ microwave-optical incompatibility problem we propose to use coded-modulation based on: (i) coded-OFDM as multiplexing technology, and (ii) to use $Q$ ary bit-interleaved coded pulse-amplitude modulation (PAM).

The paper is organized as follows. The coded-OFDM concept is introduced in Section II, while the coded-MIMO concept and space-time coding is introduced in Section III. Both concepts employ LDPC codes. Both scenarios are evaluated in terms of bit-error rates (BERs) and achievable information rates, assuming that photo-detection is non-ideal, while the atmospheric turbulence is modeled by using GammaGamma distribution function due to Al-Habash et al. [6]. The achievable information rates (the lower bound on channel capacity) are studied in Section IV. Finally, the last Section concludes the paper.

\section{CODED ORHTOGONAL FREOUENCY DIVISION MULTIPLEXING (CODED-OFDM)}

The first approach that is able to enable hybrid $\mathrm{RF} / \mathrm{microwave}$ optical communications over the atmospheric turbulent channel is based on coded-OFDM. The block diagrams of the proposed transmitter and receiver configurations are shown in Fig. 1 (a) and (b), while the transmission system based on FSO communication is shown in Fig. 1(c). The data streams from $L$ different RF channels are combined using OFDM and encoded using an LDPC encoder. The LDPC encoded data stream is then parsed into groups of $B$ bits. The $B$ bits in each group (frame) are subdivided into $K$ subgroups with the $i^{\text {th }}$ subgroup containing $b_{i}$ bits, $B=\sum b_{i}$. The $b_{i}$ bits from the $i^{\text {th }}$ subgroup are mapped into a complex-valued signal from a $2^{b_{i}}$-point signal constellation such as QAM. The complex-valued signal points from all $K$ subchannels are considered as the values of the discrete Fourier transform (DFT) of a multi-carrier OFDM signal. After D/A conversion and RF up-conversion, the OFDM signal drives a Mach-Zehnder modulator (MZM) for transmission over the FSO link. The DC component facilitates recovering the QAM symbols incoherently. At the receiver, an optical system collects the light, and focuses it onto a detector, 
which delivers an electrical signal proportional to the incoming optical power. After the RF down-conversion, carrier suppression, A/D conversion and cyclic extension removal, the transmitted signal is demodulated using the FFT algorithm. The soft outputs of the FFT demodulator are used to estimate the symbol reliabilities, which are converted to bit reliabilities, and provided as input to an LDPC iterative decoder.

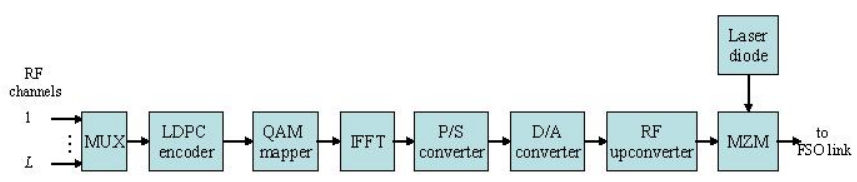

(a)

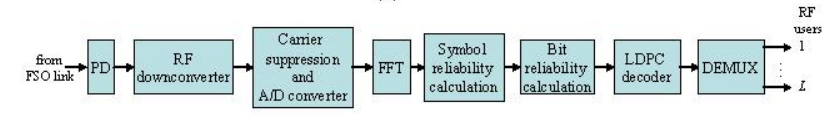

(b)

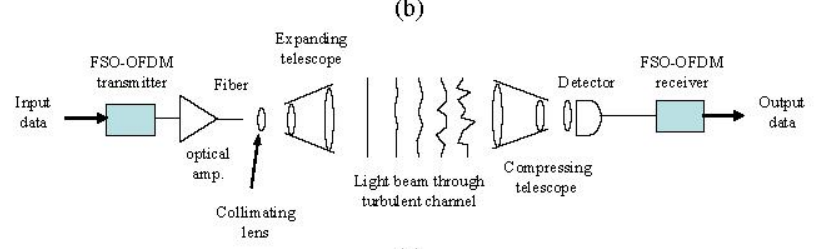

(c)

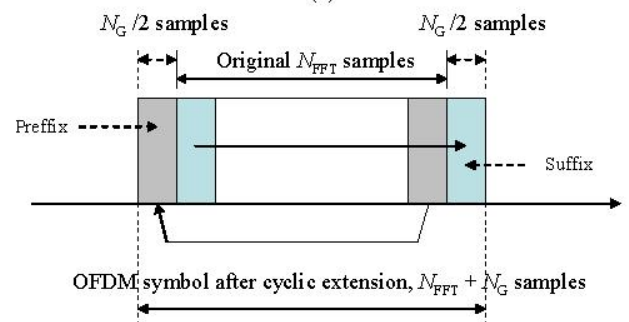

(d)

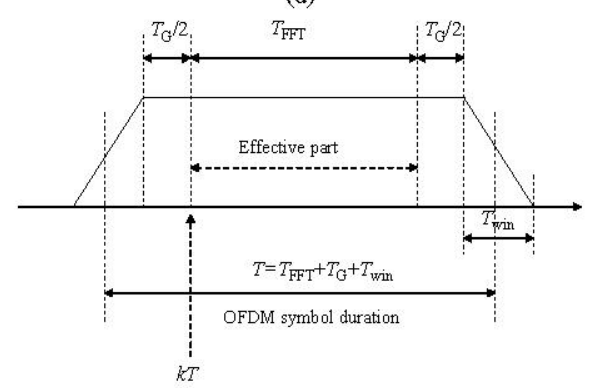

(e)

Fig. 1 LDPC-coded OFDM system: (a) transmitter configuration, (b) receiver configuration, (c) FSO link, (d) an OFDM symbol after cyclic extension, and (e) an OFDM symbol after windowing.

As mentioned above, the bipolar signals cannot be transmitted over an IM/DD link, and OFDM signals must include a DC bias in order to allow incoherent detection. The most straightforward method of DC bias addition is to add sufficient DC bias so that the resulting OFDM signal is nonnegative. This scheme is referred to as the "biased-OFDM" (BOFDM) scheme. For illustrative purposes the MZM RF input signal associated with a B-OFDM scheme is shown in Fig. 2(a). The main disadvantage of the B-OFDM scheme is its poor power efficiency. To improve the power efficiency we propose two alternative schemes.

The first alternative scheme, which we shall refer to as the "clipped-OFDM" (C-OFDM) scheme, is based on single-side band (SSB) transmission and clipping of the OFDM signal after adding a bias. The clipping can be either symmetric or asymmetric. Our initial studies have shown that in symmetric clipping the optimum bias should be selected such that $\sim 50 \%$ of the total electrical signal energy before clipping is allocated for transmission of a carrier. The MZM RF input signal for the clipped-OFDM scheme is shown in Fig. 2(b). We note that clipping introduces inter-modulation distortion that may degrade BER performance. However, because C-OFDM allocates more energy per information bit than B-OFDM a tradeoff results. The optimum choice of system parameters and their dependence on FSO channel conditions is an important issue, however, due to space limitations this study will be omitted.

In order to avoid distortion due to clipping at the transmitter, the information-bearing signal may be mapped into the optical domain by modulating the electrical field of the optical carrier using a $\mathrm{LiNbO}_{3} \mathrm{MZM}$. In this case, the clipping will be performed by the receiver through the squaring operation inherent in the measurement of optical intensity (by photodetector). The distortion introduced by photodetector may be reduced by proper filtering. Notice that the U-OFDM scheme will be less power-efficient that the $\mathrm{C}$ OFDM scheme, but is still expected to be better than the BOFDM scheme. The MZM RF input signal for the U-OFDM scheme is shown in Fig. 2(c).

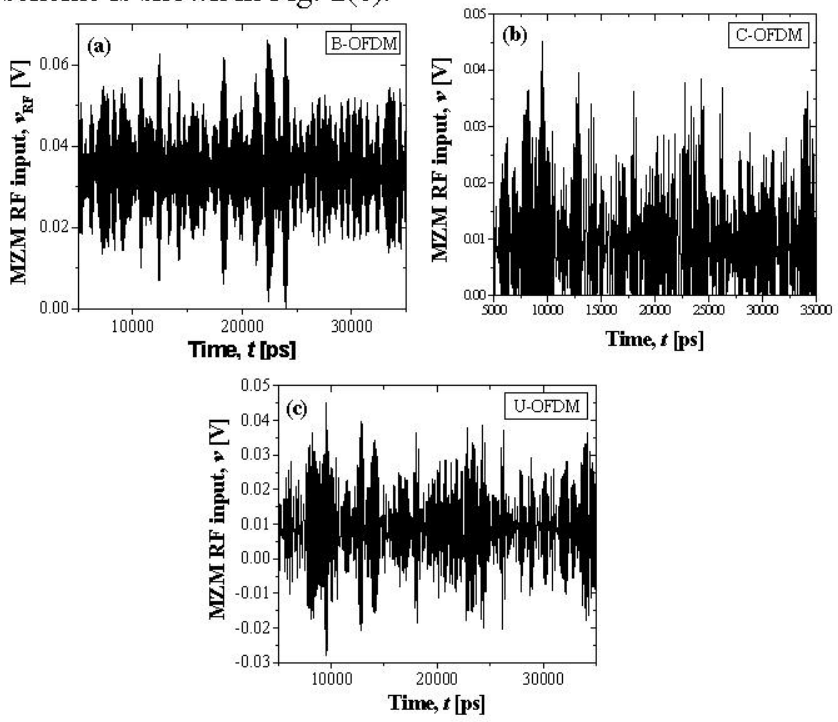

Fig. 2 Waveforms of the SSB OFDM signal with 64 sub-carriers at MZM RF input in a back-to-back configuration for: (a) B-OFDM, (b) C-OFDM, and (c) U-OFDM.

The influence of both atmospheric turbulence and receiver electronic noise (AWGN) on QPSK and 16-QAM SSB FSOOFDM systems is illustrated in Fig. 3. Results obtained from a SSB OFDM system with 64 sub-carriers are shown. The average launched power is set to $0 \mathrm{dBm}$, the electrical SNR is 
set to $18 \mathrm{~dB}$, and the received signal constellation diagrams are obtained assuming weak atmospheric turbulence. We note that turbulent propagation changes the symmetry of these signal clusters from circular (i.e., for a pure AWGN channel) to elliptical (see Fig. 3) for the FSO channel. Both C-OFDM and U-OFDM schemes are more immune to atmospheric turbulence than is the B-OFDM scheme. The U-OFDM system performs only slightly better than C-OFDM. It appears that the better power efficiency of C-OFDM compensates the distortion introduced by clipping. Higher energy per bit associated with C-OFDM may result in improved immunity to electric noise. Higher immunity to electrical noise may result in slightly better BER performance of C-OFDM scheme when compared to U-OFDM scheme.

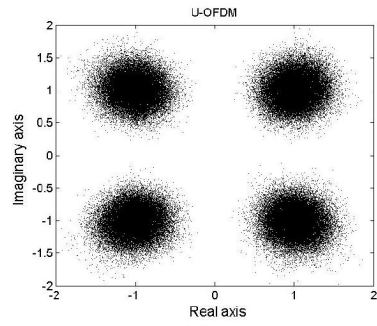

(a)

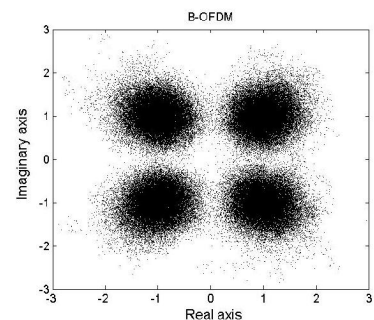

(c)

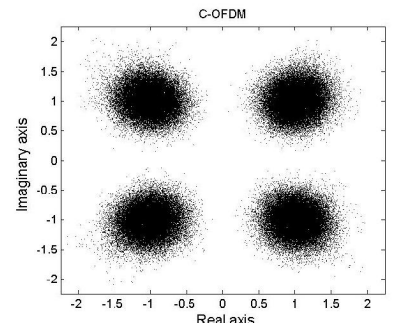

(b)

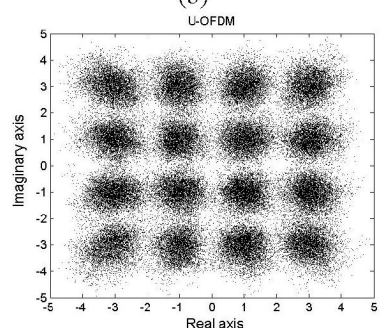

(d)
Fig. 3 Received constellation diagrams of QPSK (a)-(c) and 16-QAM (d) SSB FSO-OFDM systems with electrical SNR per bit of $18 \mathrm{~dB}$ under the weak turbulence for: (a),(d) U-OFDM scheme, (b) C-OFDM scheme, and (c) BOFDM scheme.

High-speed optical receivers commonly employ the transimpedance design, which is a good compromise between noise and bandwidth. The PIN photodiode output current can be written as

$$
\begin{aligned}
& i(t)=\mathrm{R}\left|\left(s_{\mathrm{OFDM}}(t)+b\right) * h(t)\right|^{2}= \\
& \mathrm{R}\left[\left|s_{\mathrm{OFDM}}(t) * h(t)\right|^{2}+|b * h(t)|^{2}+2 R_{e}\left\{\left(s_{\mathrm{OFDM}}(t) * h(t)\right)(b * h(t))\right\}\right],
\end{aligned}
$$

where $s_{\text {OFDM }}(t)$ denotes the transmitted OFDM signal in RF domain, upon D/A conversion and RF up-conversion, defined by

$$
\begin{gathered}
s_{\mathrm{OFDM}}(t)=\operatorname{Re}\left\{\sum_{k=-\infty}^{\infty} w(t-k T) \sum_{i=-\left(N_{\mathrm{FFT}} / 2\right)}^{\left(N_{\mathrm{FFT}} / 2\right)-1} X_{i, k} \cdot e^{j 2 \pi \frac{i}{T_{F F T}} \cdot(t-k T)} e^{j 2 \pi f_{R F} t}\right\} \\
k T-\left(T_{\mathrm{G}} / 2\right)-T_{\text {win }} \leq t \leq k T+T_{\mathrm{FFT}}+\left(T_{\mathrm{G}} / 2\right)+T_{\text {win }}
\end{gathered}
$$

$b$ is the DC bias component, and $R$ denotes the photodiode responsivity. In (2) $X_{i, k}$ denotes the $i$-th sub-carrier QAM symbol of the $k$-th OFDM symbol, $T$ denotes the OFDM symbol duration, $T_{\mathrm{FFT}}$ is the FFT part duration, $T_{\mathrm{G}}$ is the guard interval (cyclic extension) duration, $T_{\text {win }}$ denotes the windowing interval duration, $w(t)$ is the window function, and $f_{\mathrm{RF}}$ denotes the RF carrier frequency.

Simulation results of an LDPC coded SSB U-OFDM system under the strong turbulence regime are given in Fig. 4(a). The coding gain improvement of the LDPC-coded OFDM system over the LDPC-coded OOK system is $20.24 \mathrm{~dB}$ for QPSK, and $23.38 \mathrm{~dB}$ for BPSK. The 16-QAM FSOOFDM system is not able to operate in the regime of strong turbulence. The comparison of different LDPC coded SSB OFDM schemes in weak turbulence $\left(\sigma_{R}=0.6\right)$, is given in Fig. 4(b). The C-OFDM scheme slightly outperforms the U-OFDM scheme. Both C-OFDM and U-OFDM schemes outperform the B-OFDM scheme by approximately $1.5 \mathrm{~dB}$ at BER of $10^{-5}$.

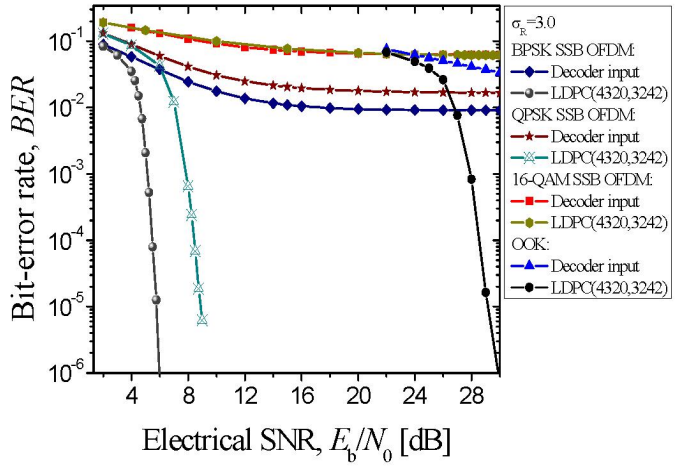

(a)

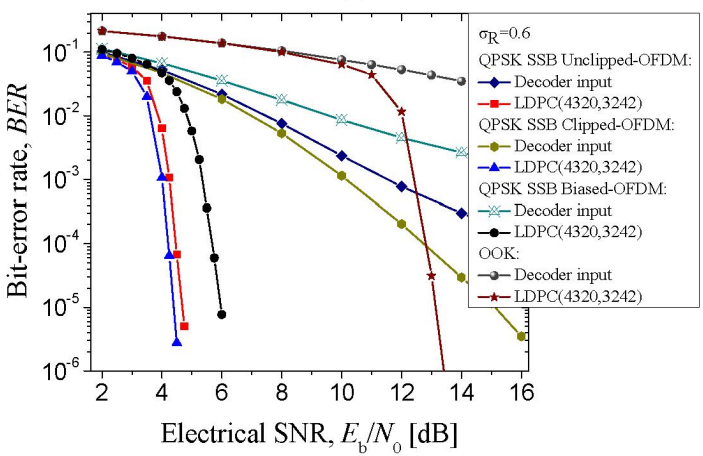

(b)

Fig. 4 (a) BER performance of LDPC-coded SSB U-OFDM system with 64subcarriers under the strong turbulence. Block-circulant LDPC code (4320,3242) of rate 0.75 is employed. (b) Comparison of different LDPC coded SSB FSO-OFDM systems with 64-subcarriers under the weak turbulence.

The numerical results shown in Figs. 3-4 are obtained adopting the Gamma-Gamma probability density function (PDF) [6]

$$
f(I)=\frac{2(\alpha \beta)^{(\alpha+\beta) / 2}}{\Gamma(\alpha) \Gamma(\beta)} I^{(\alpha+\beta) / 2-1} K_{\alpha-\beta}(2 \sqrt{\alpha \beta I}), I>0
$$

where $\alpha$ and $\beta$ are PDF parameters describing the scintillation experienced by plane waves, and in the case of zero-inner scale are given by: 
$\alpha=\left[\exp \left(\frac{0.49 \sigma_{R}^{2}}{\left(1+1.11 \sigma_{R}^{1 / 5}\right)^{7 / 6}}\right)-1\right]^{-1}, \beta=\left[\exp \left(\frac{0.51 \sigma_{R}^{2}}{\left(1+0.69 \sigma_{R}^{12 / 5}\right)^{5 / 6}}\right)-1\right]^{-1}$

To quantify the strength of the turbulence we used the unitless Rytov variance, given by

$$
\sigma_{R}^{2}=1.23 C_{n}^{2} k^{7 / 6} L^{11 / 6},
$$

where $k=2 \pi / \lambda$ is the optical wave number, $\lambda$ is the wavelength, $L$ is propagation distance, and $C_{n}^{2}$ is the refractive index structure parameter, which we assume to be constant for horizontal paths. This model is valid for a wide range of atmospheric turbulence conditions, ranging from weak to strong.

For the results shown in Figs. 3-4, the received intensity samples are considered to be independent and uncorrelated. In reality, especially at high bit rates, the channel has temporal correlation, and consecutive bits that propagate experience similar channel conditions. In many OFDM systems this approach is reasonable for the following reasons: (i) when the channel conditions do not vary, a simple channel estimation techniques based on pilot signals can be used to overcome the temporal correlation, and (ii) the immunity to temporal correlation can further be improved by using interleaving. The interleaving can be visualized as the forming an $L \mathrm{x} n$ ( $n$ is the codeword length) array of $L$ LDPC codewords (the parameter $L$ is known as interleaving degree) written row by row, and transmitting the array entries column by column. If the original code can correct a single error burst of length $l$ or less, then the interleaved code can correct a single error burst of length $l L$. Therefore, interleaved OFDM can successfully eliminate temporal correlation introduced by the FSO channel. To illustrate the applicability of LDPC-coded OFDM in the presence of temporal correlation we performed simulations by employing the joint temporal correlative distribution model similar to that reported in [7], which describes the fading in an FSO channel at a single point of space at multiple instances of time. This method is based on the Rytov method to derive the normalized log-amplitude covariance function for two positions in a receiving plane perpendicular to the direction of propagation [7]:

$$
b_{X}\left(d_{i j}\right)=\frac{B_{X}\left(P_{i}, P_{j}\right)}{B_{X}\left(P_{i}, P_{i}\right)},
$$

where $d_{i j}$ is the distance between points $P_{i}$ and $P_{j} . B_{X}$ denotes the log-amplitude $X$ covariance function:

$$
B_{X}\left(P_{i}, P_{j}\right)=E\left[X\left(P_{i}\right) X\left(P_{j}\right)\right]-E\left[X\left(P_{i}\right)\right] E\left[X\left(P_{j}\right)\right],
$$

and it is found to be exponential for both plane and spherical waves [8] under the weak turbulence

$$
b_{X}(\tau)=\exp \left(-\left(\frac{|\tau|}{\tau_{0}}\right)^{5 / 3}\right),
$$

where $\tau_{0}$ is the coherence time, with typical values being in the range from $10 \mu \mathrm{s}$ to $10 \mathrm{~ms}$. The corresponding correlation matrix, based on [7] and Eq. (10), can be written as

$$
C_{X}=\left[\begin{array}{cccc}
\sigma_{\mathrm{X}}^{2} & \sigma_{\mathrm{X}}^{2} b_{X}\left(\frac{T}{\tau_{0}} d_{0}\right) & \ldots & \sigma_{\mathrm{X}}^{2} b_{X}\left(\frac{(n-1) T}{\tau_{0}} d_{0}\right) \\
\sigma_{\mathrm{X}}^{2} b_{X}\left(\frac{T}{\tau_{0}} d_{0}\right) & \sigma_{\mathrm{X}}^{2} & \ldots & \sigma_{\mathrm{X}}^{2} b_{X}\left(\frac{(n-2) T}{\tau_{0}} d_{0}\right) \\
\ldots & \ldots & \ldots & \ldots \\
\sigma_{\mathrm{X}}^{2} b_{X}\left(\frac{(n-1) T}{\tau_{0}} d_{0}\right) & \sigma_{\mathrm{X}}^{2} b_{X}\left(\frac{(n-2) T}{\tau_{0}} d_{0}\right) & \ldots & \sigma_{\mathrm{x}}^{2}
\end{array}\right]
$$

$\sigma_{X}^{2}$ in (11) denotes the variance of the log-normally distributed amplitude, which for plane wave can be approximated as [8]

$$
\sigma_{X}^{2} \cong 0.56 k^{7 / 6} \int_{0}^{L} C_{n}^{2}(x)(L-x)^{5 / 6} d x,
$$

where the wave number $k$, propagation length $L$, and the refractive index structure parameter $C_{n}^{2}$ were introduced earlier. $T$ is the time interval between observations, which corresponds to the OFDM symbol period. The results of simulations using the model described by Eqs. (10)-(12) are shown in Fig. 5. The standard deviation $\sigma_{X}$ is set to 0.6 (notice that $\sigma_{\mathrm{X}}$ is different from Rytov standard deviation $\sigma_{\mathrm{R}}$ used earlier, and for horizontal paths $\sigma_{X} \sim 0.498 \sigma_{R}$ ). The BER performance can further be improved by using the interleaver with larger interleaving degree than that used in Fig. 5, at the expense of increasing encoder/decoder complexity. Notice the on-off keying (OOK) modulation scheme enters BER floor for this value of standard deviation $\left(\sigma_{X}=0.6\right)$, and even advanced FEC is not able to help too much. However, LDPC-coded OOK is able to operate properly at lower standard deviations $\sigma_{\mathrm{X}}$. To generate temporally correlated samples we used two different methods, the first one is based on the LevinsonDurbin algorithm [9], and the second one is based on an algorithm due to Wood and Chan [10]. Both methods gave identical plots.

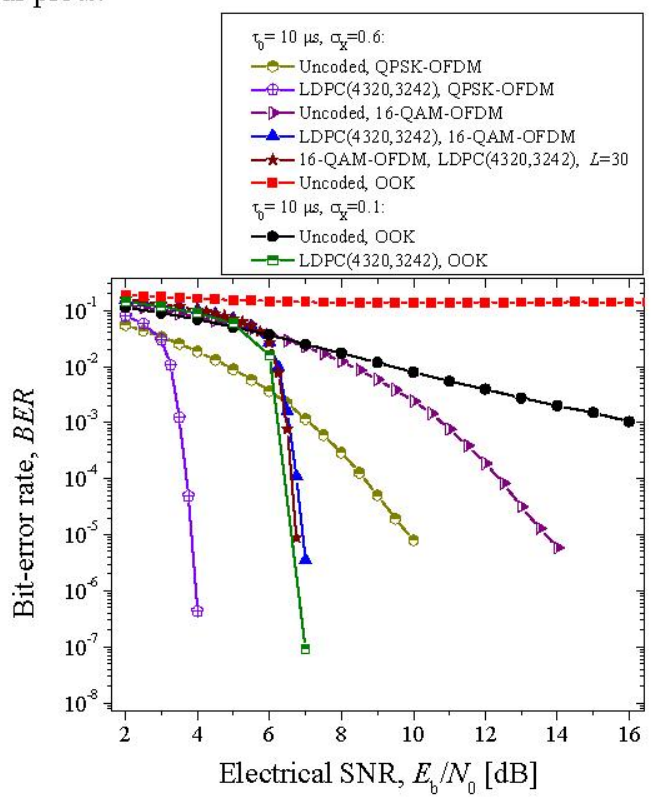

Fig. 5 BER performance in the presence of temporal correlation 


\section{CODED-MIMO AND SPACE-TIME CODING}

The second approach enabling the communication over strong atmospheric turbulent channel, the coded-MIMO concept is illustrated in Fig. 6. $M$ optical sources are all pointed toward the distant array of $N$ photodetectors using an expanding telescope, as shown in Fig. 6(a). We assume that the beam spots on the receiver side are sufficiently wide to illuminate a whole photodetector array (see Fig. 6(b)). The information bearing signal is LDPC encoded. A space-time (ST) encoder accepts $K$ encoded bits $x_{k}(k=1,2, \ldots, K)$ from an LDPC encoder. The ST encoder maps the input bits into the TxM matrix $\mathbf{O}$ whose entries are chosen from

$$
\left\{x_{1}, x_{2}, \ldots, x_{K}, \bar{x}_{1}, \bar{x}_{2}, \ldots, \bar{x}_{K}\right\}
$$

so that the separation of decision statistics is possible at the receiver side (with $\bar{x}$ we denoted the complement of $x, 1-x$ ). For example, the ST code with $K=T=M=4$ based on orthogonal designs [11] is given below

$$
O=\left[\begin{array}{llll}
x_{1} & x_{2} & x_{3} & x_{4} \\
\bar{x}_{2} & x_{1} & \bar{x}_{4} & x_{3} \\
\bar{x}_{3} & x_{4} & x_{1} & \bar{x}_{2} \\
\bar{x}_{4} & \bar{x}_{3} & x_{2} & x_{1}
\end{array}\right]
$$

It can easily be verified that for FSO systems, ST codes from orthogonal designs do not satisfy the orthogonality property [11]

$$
\operatorname{det}\left(O O^{T}\right)=\left[\sum_{i} x_{i}^{2}\right]^{n}
$$

Nevertheless the separation of decision statistics can be achieved, when the complements are properly chosen so that a simple a posteriori probability (APP) ST decoder still exists. Note that given the lack of orthogonality, $O$ is not optimum. The LLR, calculated in ST soft-decoding block (see Fig. 6(c)), corresponding to transmitted bit $x_{k}$ for example above, can be determined by:

$L\left(x_{k}\right)=\sum_{n=1}^{N}\left[\frac{\left(\tilde{x}_{k}-1\right)^{2}}{N_{0}}+\frac{I_{n 1}^{2}+I_{n 2}^{2}+I_{n 3}^{2}+I_{n 4}^{2}-1}{N_{0}}-\frac{\tilde{x}_{k}^{2}}{N_{0}}\right] ; k=1, \ldots, 4$

where

$$
\begin{aligned}
& \tilde{x}_{1}=\sum_{n=1}^{N}\left(I_{n 1} y_{n 1}+I_{n 2} y_{n 2}+I_{n 3} y_{n 3}+I_{n 4} y_{n 4}-I_{n 1} I_{n 2}-I_{n 2} I_{n 3}-I_{n 1} I_{n 3}-I_{n 3} I_{n 4}-I_{n 1} I_{n 4}-I_{n 2} I_{n 4}\right) \\
& \tilde{x}_{2}=\sum_{n=1}^{N}\left(I_{n 2} y_{n 1}-I_{n 1} y_{n 2}-I_{n 4} y_{n 3}+I_{n 3} y_{4}+I_{n 1}^{2}+I_{n 1} I_{n 3}+I_{n 1} I_{n 4}+I_{n 4}^{2}-I_{n 1} I_{n 3}-I_{n 2} I_{n 3}\right) \\
& \tilde{x}_{3}=\sum_{n=1}^{N}\left(I_{n 3} y_{n 1}+I_{n 4} y_{n 2}-I_{n 1} y_{n 3}-I_{n 2} y_{n 4}-I_{n 1} I_{n 4}-I_{n 3} I_{n 4}+I_{n 1}^{2}+I_{n 1} I_{n 4}+I_{n 1} I_{n 2}+I_{n 2}^{2}\right) \\
& \tilde{x}_{4}=\sum_{n=1}^{N}\left(I_{n 4} y_{n 1}-I_{n 3} y_{n 2}+I_{n 2} y_{n 3}-I_{n 1} y_{n 4}+I_{n 1} I_{n 3}+I_{n 3}^{2}-I_{n 1} I_{n 2}-I_{n 2} I_{n 4}+I_{n 1}^{2}+I_{n 1} I_{n 2}\right)
\end{aligned}
$$

In (15)-(16) $N_{0} / 2$ represents the double-sided power spectral density of TA thermal noise (that is white Gaussian), and $I_{n m}$ represents the channel intensity coefficient between $n$th photodetector and $m$ th light source, described by GammaGamma PDF introduced in (3). Corresponding repetitionMIMO scheme is obtained by repeating the first row in (13) additional three times.

In order to enable hybrid $\mathrm{RF} /$ microwave-optical communication over the atmospheric turbulence channel we propose to combine LDPC-coded MIMO with an appropriate multilevel modulation scheme such as Q-ary pulse-position modulation (PPM) or Q-ary pulse-amplitude modulation (PAM), which is considered here. The bit streams originating from $L \mathrm{RF} /$ microwave sources are multiplexed and encoded using an $(n, k)$ LDPC code of code rate $r=k / n$ ( $k$-the number of information bits, $n$-the codeword length). The $m \times n$ blockinterleaver, collects $m$ code-words written row-wise. The mapper accepts $m$ bits from the interleaver column-wise and determines the corresponding symbol for Q-ary $\left(Q=2^{m}\right)$ PAM signaling using a Gray mapping rule. The optical transmitter and receiver configurations are shown in Figs. 7(a) and 7(b), respectively. By using this scheme, information bits from the same source are allocated into different PAM symbols. Therefore, this technique improves the tolerance to atmospheric turbulence, because different Q-ary PAM symbols experience different atmospheric turbulence conditions. The output of the optical receiver is processed to determine the symbol reliabilities:

$$
\lambda(q)=-\sum_{n=1}^{N} \frac{\left(y_{n}-\operatorname{map}(q) d \sum_{m=1}^{M} I_{n m}\right)^{2}}{N_{0}} ; \quad q=0,1, \ldots, Q-1
$$

where with map $(q)$ we denoted the corresponding mapping rule. In (17) $y_{n}(n=1,2, \ldots, N)$ represents the output of $n$th receiver in MIMO configuration, $d$ is separation between two neighboring constellation points, while $N_{0}$ and $I_{n m}$ are introduced earlier. The bit reliabilities are determined from these symbol reliabilities by

$$
L\left(c_{i}\right)=\log \frac{\sum_{\boldsymbol{c} c_{i}=0} \exp [\lambda(q)] \exp \left(\sum_{\boldsymbol{c} c_{j}=0, j \neq i} L_{a}\left(c_{j}\right)\right)}{\sum_{\boldsymbol{c} c_{i}=1} \exp [\lambda(q)] \exp \left(\sum_{\boldsymbol{c} c c_{j}=0, j \neq i} L_{a}\left(c_{j}\right)\right)} .
$$

The LDPC decoder hard decisions are demultiplexed and delivered to different $\mathrm{RF} /$ microwave users. In (18) with $L_{a}\left(c_{j}\right)$ we denoted a priori information determined from the LDPC decoder extrinsic LLRs. $c_{j}$ represents the $j$ th bit in an observed symbol $q$ binary representation $c=\left(c_{1}, c_{2}, \ldots, c_{l}\right)$.

The basis function for Q-ary PAM is given by

$\phi_{\mathrm{PAM}}(t)=\frac{1}{\sqrt{T}} \operatorname{rect}(t / T), \quad \operatorname{rect}(t)=\left\{\begin{array}{cc}1, & 0 \leq t<1 \\ 0, & \text { otherwise }\end{array}\right.$

while the signal constellation points by $A_{q}=q d(q=0,1, \ldots, Q-1)$. The average symbol energy is given by

$$
E_{s}=\frac{(Q-1)(2 Q-1)}{6} d^{2}
$$

and it is related to the bit-energy $E_{\mathrm{b}}$ by $E_{\mathrm{s}}=E_{\mathrm{b}} \log _{2} Q$.

Other multilevel schemes, for example quadrature amplitude-modulation (QAM), are also applicable. In QAM the use of additional DC bias is required because negative signals cannot be transmitted over an IM/DD system, resulting in poor power efficiency.

The results of simulations for bit-interleaved LDPC $(6419,4794)$-coded PAM are shown in Fig. 8 for different MIMO configurations and different number of signal constellation points employing the Gray mapping rule. The excellent BER performance improvement over single optical source-single photodetector case is obtained (about $23 \mathrm{~dB}$ for $M=N=4, Q=4$ over $M=N=1, Q=4$ ). 


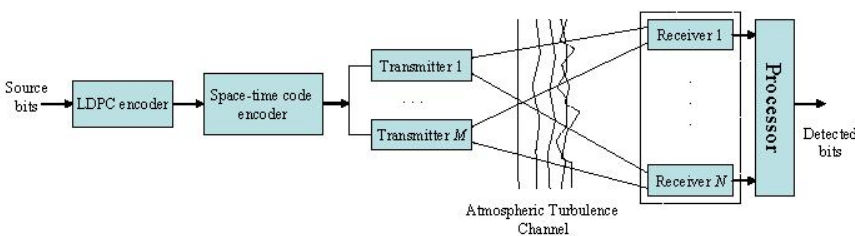

(a)

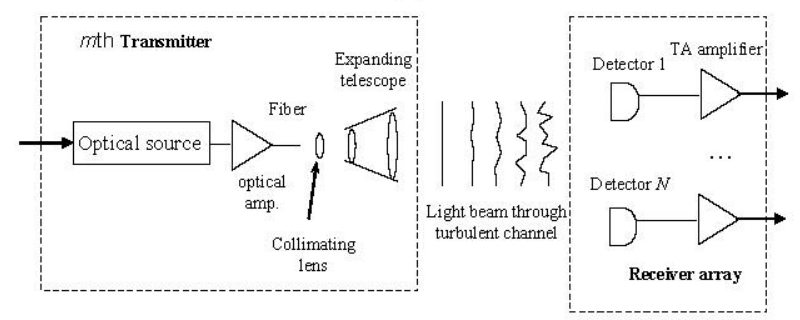

(b)

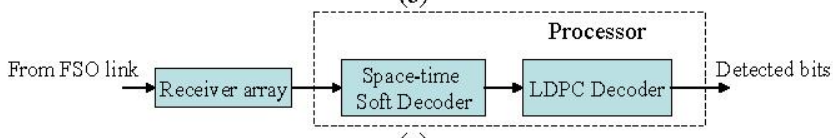

(c)

Fig. 6 (a) Atmospheric optical LDPC-coded MIMO system with space-time block codes, (b) $m$ th transmitter and receiver array configurations, and (c) processor configuration.

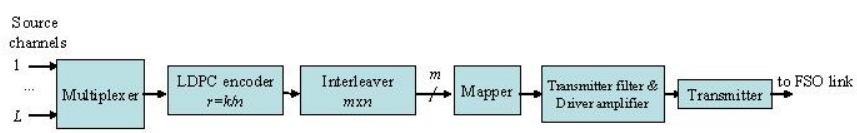

(a)

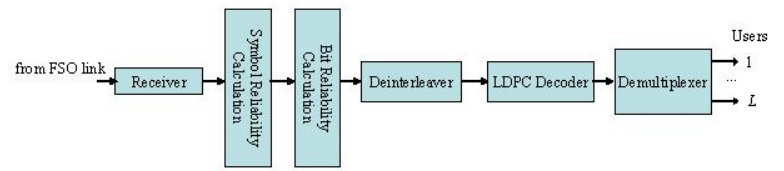

(b)

Fig. 7 Hybrid RF/microwave-optical communication over the atmospheric turbulent channel using BI LDPC-coded Q-ary PAM: (a) transmitter side, (b) receiver side.

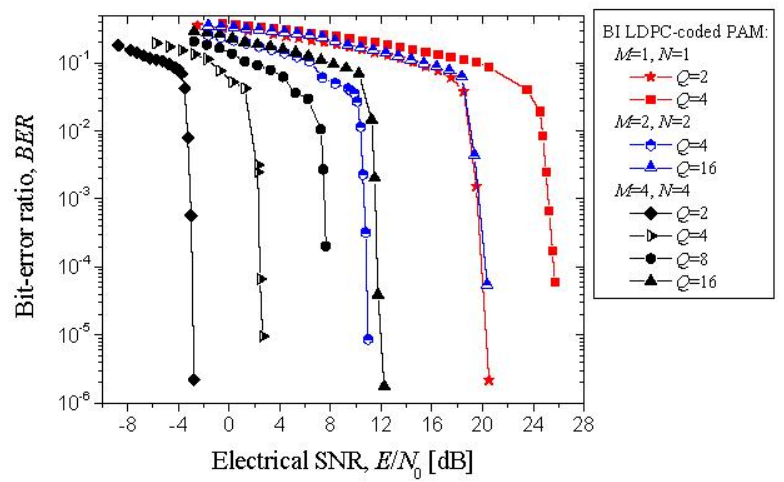

Fig. 8 BER performance of BI-LDPC $(6419,4794)$-coded PAM with repetition MIMO.

The comparison between ST-coded MIMO and repetition MIMO is given in Fig. 9. The Alamouti-like ST code performance is comparable to the repetition MIMO, while $T=4$ ST performs worse than the corresponding repetition MIMO. The reason for such a behavior comes from the fact that we operate with non-negative real signals rather than with complex, so that the space-time codes from orthogonal designs are not optimal in an FSO channel. The LDPC-coded MIMO with Alamouti-like code $(M=2)$ and $N=4$ photodetectors provides about $20 \mathrm{~dB}$ improvement over LDPC-coded OOK with single optical source and single photodetector. The BER vs. electrical SNR plots in Figs. 8-9 are obtained assuming the strong turbulence regime ( $\left.\sigma_{\mathrm{R}}=3.0, \alpha=5.485, \beta=1.1156\right)$, while the SNR is defined on a receiver side (observed in a back-toback configuration).

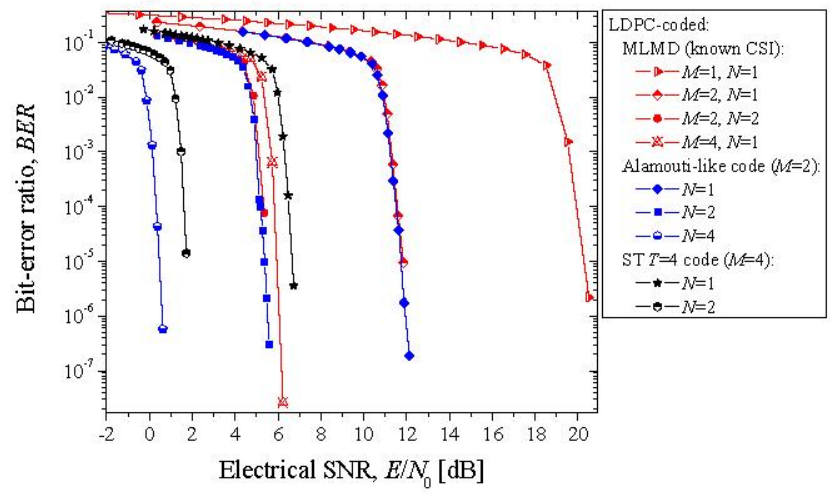

Fig. 9 BERs of binary LDPC $(6419,4794)$-coded MIMO ST coding scheme against LDPC-coded repetition MIMO.

\section{ACHIEVABLE INFORMATION RATE STUDY}

The numerical results for LDPC-coded MIMO with/without ST coding were reported in previous Section. Both schemes provide excellent coding gains when compared with single optical source - single photodetector case, so that naturally arises the question how much closely can channel capacity be approached using those two schemes. We turn now our attention to the calculation of achievable information rates. We determine the MIMO i.i.d capacity $R$ (also known as achievable information rate) for Q-ary PAM by using Eq. (5) in [12] as follows

$$
R=\log _{2} Q-\underset{I_{n}}{E} \underset{\boldsymbol{y} \mid I_{n}}{E}\left\{\log _{2}\left[1+\sum_{q=1}^{Q-1} \exp \left(-\sum_{n=1}^{N} \frac{\left(Y_{n, q}-q A I\right)^{2}-Y_{n, q=0}^{2}}{N_{0}}\right)\right]\right\},
$$

where $I_{n}=\sum_{m=1}^{M} I_{n, m}$, and $Y_{n, q}$ denotes the $n$th receiver response to $q$ th symbol (other parameters are introduced earlier). Notice that ensemble averaging is to be done for different channel conditions $\left(I_{n}\right)$ and for different thermal noise realizations $\left(\boldsymbol{y} \mid I_{n}\right.$; $\left.\boldsymbol{y}=\left(y_{1}, \ldots, y_{N}\right)\right)$ by using Monte Carlo simulations. In Fig. 10(a) we plotted the i.i.d. capacity for binary transmission in strong turbulence regime $\left(\sigma_{\mathrm{R}}=3.0\right)$ for different number of optical sources, and photodetectors, against the electrical signal to noise ratio per photodetector in the presence of scintillation. The parameters $\alpha$ and $\beta$ corresponding to $\sigma_{\mathrm{R}}=3.0$ are $\alpha=5.485$, $\beta=1.1156$. Slightly better improvement is obtained by increasing the number of optical sources than increasing the number of photodetectors. The MIMO FSO systems with $M=N=2$ and $M=4, N=1$ are comparable. In Fig. 10(b) we 
plotted the i.i.d. capacity for $Q$-ary pulse-amplitude modulation. Significant i.i.d. channel capacity improvement is obtained by employing the MIMO concept.

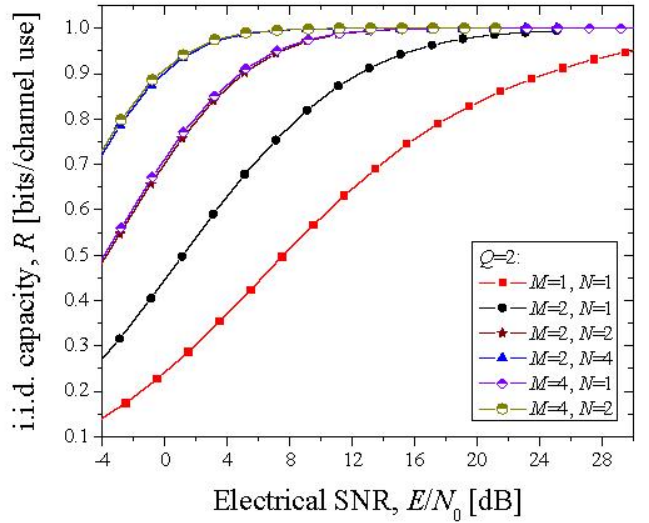

(a)

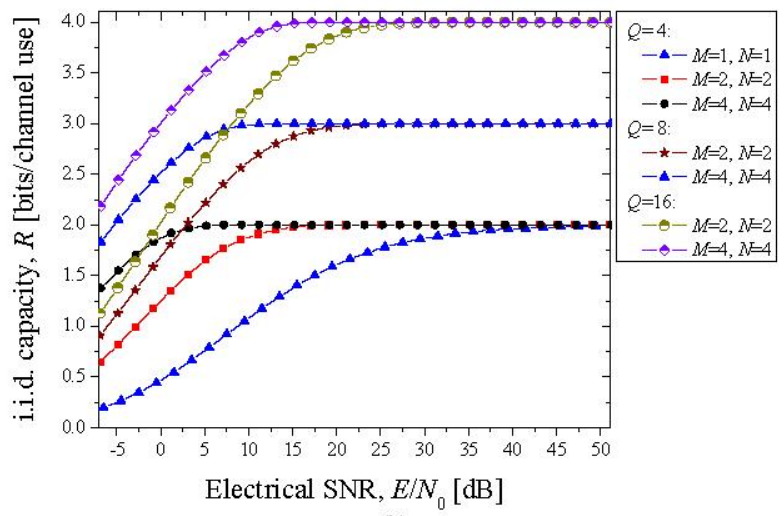

(b)

Fig. 10 i.i.d channel capacity for different numbers of optical sources $(M)$, and photodetectors $(N)$ in strong turbulence regime $\left(\sigma_{\mathrm{R}}=3.0\right)$ for: (a) binary transmission, and (b) $Q$-ary PAM.

\section{CONCLUSION}

In this paper, we proposed two alternative schemes that can be used to enable communication over the strong turbulence channel: (i) coded-OFDM, and (ii) coded-MIMO concept. The coding scheme employed in both concepts is based on structured LDPC codes. The bit-error rates and achievable information rates are reported assuming non-ideal photodetection. The Alamouti-like based LDPC-coded MIMO scheme with four photodetectors provides about $20 \mathrm{~dB}$ improvement over single optical source - single photodetector scheme at BER of $10^{-6}$. LDPC-coded OFDM based on SSB UOFDM and BPSK provides more than $23 \mathrm{~dB}$ improvement over LDPC-coded OOK when operated in strong turbulence regime.
To enable hybrid $\mathrm{RF} /$ microwave-optical communication over the atmospheric turbulent channel we proposed the use of following two concepts: (i) the use of coded-OFDM as multiplexing technique, and (ii) the concept of bit-interleaved LDPC-coded modulation based on Q-ary PAM.

Although excellent BER performance improvements, even $20 \mathrm{~dB}$ improvement over LDPC-coded OOK when Alamutilike scheme is used in strong turbulence regime, from the channel capacity studies we can conclude that that we are still several dBs away from channel capacity curves. Novel spacetime coding approaches, taking into account the physics of a FSO channel into account, are required which was left for further research. For the description of LDPC codes employed in this paper an interested reader is referred to [2]-[3]

\section{ACKNOWLEDGMENTS}

The author would like to thank S. Denic, B. Vasic, M. A. Neifeld and J. Anguita from University of Arizona for their involvement in earlier joint work on free-space optical communication systems.

\section{REFERENCES}

[1] H. Willebrand, B.S. Ghuman, Free-Space Optics: Enabling Optical Connectivity in Today's Networks. Indianapolis, Indiana: Sams Publishing, 2002.

[2] I. B. Djordjevic, B. Vasic, M. A. Neifeld, "LDPC coded OFDM over the atmospheric turbulence channel," Optics Express, vol. 15, no. 10, pp. 6332-6346, May 2007.

[3] I. B. Djordjevic, B. Vasic, M. A. Neifeld, "LDPC-coded OFDM for optical communication systems with direct detection," IEEE/LEOS J. Sel. Top. Qucntum Electron., vol. 13, no. 5, pp. 1446 - 1454, Sept.-Oct. 2007

[4] I. B. Djordjevic, B. Vasic, M. A. Neifeld, "LDPC coded orthogonal frequency division multiplexing over the atmospheric turbulence channel," in Proc. CLEO/OELS 2006, May 21-26, 2006, Long Beach, California, Paper no. CMDD5. (Invited paper.)

[5] I. B. Djordjevic, S. Denic, J. Anguita, B. Vasic, and M. A. Neifeld, "LDPC-coded MIMO optical communication over the atmospheric turbulence channel," accepted for presentation at Globecom 2007.

[6] M.A. Al-Habash, L.C. Andrews, R.L. Phillips, "Mathematical model for the irradiance probability density function of a laser beam propagating through turbulent media," Opt. Engineering, vol. 40, 1554-1562, 2001.

[7] X. Zhu, and J.M. Kahn, "Free-space optical communication through atmospheric turbulence channels," IEEE Trans. Commun, vol. 50, pp. $1293-1300,2002$

[8] L. C. Andrews, R. L. Philips, Laser Beam Propagation Through Random Media: SPIE Optical Engineering Press, 1998.

[9] J. Durbin, "Efficient estimation of parameters in moving-average models," Biometrica, vol. 46, pp. 306-316, 1959.

[10] A. T. A Wood, and G. Chan, "Simulation of stationary Gaussian processes in [0,1]d," J. Comp. Groph. Stot., vol. 3, pp. 409-432, 1994.

[11] V. Tarokh, H. Jafarkani, A. R. Calderbank, "Space-time block codes from orthogonal designs," IEEE Trans. Inform. Theory, vol. 45, no. 5, pp. 1456-1467, Jul. 1999.

[12] G. Ungerboeck, "Channel coding with multilevel/phase signals," IEEE Trans. Inform. Theory, vol. 28, pp. 55-67, Jan. 1982 